\title{
COMPARISON OF THE DIGESTIBILITY OF DIETS BASED ON FISH MEAL AND SOYBEAN MEAL IN Litopenaeus vannamei BOONE 1931, USING DIFFERENT TEMPERATURES AND SALINITIES FOR CULTURE \\ COMPARACIÓN DE LA DIGESTIBILIDAD DE DIETAS A BASE DE HARINA DE PESCADO Y HARINA DE SOYA EN Litopenaeus vannamei BOONE 1931, UTILIZANDO DIFERENTES TEMPERATURAS Y SALINIDADES DE CULTIVO
}

\author{
Héctor Cabanillas-Beltrán ${ }^{1}$ \\ Jesús T. Ponce-Palafox ${ }^{2}$ \\ Carlos A. Martínez-Palacios ${ }^{3}$ \\ Ma. Cristina Chávez-Sánchez ${ }^{4}$ \\ Lindsay G. Ross ${ }^{5}$ \\ ${ }^{1}$ Instituto Tecnológico de Tepic \\ Av. Tecnológico 2595 \\ Tepic CP 63175, Nayarit, México \\ ${ }^{2}$ Centro de Investigaciones Biológicas \\ Universidad Autónoma del Estado de Morelos \\ Morelos, México \\ ${ }^{3}$ Instituto de Investigaciones sobre Recursos Naturales \\ UMSNH, Morelia, Michoacán, México \\ Av. San Juanito Itzicuaro $\mathbf{s} / \mathbf{n}$ \\ Col. San Juanito Itzicuaro \\ Morelia CP 58330, México \\ ${ }^{4}$ Unidad Mazatlán en Acuicultura y Manejo Ambiental del CIAD, A.C. \\ Av. Sábalo Cerritos s/n \\ Estero del Yugo \\ Mazatlán CP 82010, Sinaloa, México \\ ${ }^{5}$ Institute of Aquaculture \\ University of Stirling \\ Stirling FK9-4LA, Scotland, UK \\ Recibido en diciembre de 2000; aceptado en septiembre de 2001
}


Ciencias Marinas, Vol. 27, No. 4, 2001

\begin{abstract}
The digestibility of fish meal and soybean meal by Litopenaeus vannamei was determined at different temperatures $\left(22^{\circ} \mathrm{C}\right.$ and $\left.28^{\circ} \mathrm{C}\right)$ and salinities $(16 \%$ and $35 \%$ ) using practical diets based on ingredients available in Mexico. The digestibility of soybean meal was consistently higher compared to that of fish meal, in terms of the apparent digestibilities of dry matter, crude protein and aminoacids, suggesting that soybean meal proteins are more readily digested. For fish meal, the apparent digestibility of the dry matter was reduced by high salinity at $28^{\circ} \mathrm{C}$, and the carbohydrate apparent digestibility was reduced by high salinity at both temperatures. The high salinity also reduced the digestibility of dry-matter, lipids and carbohydrates in soybean meal at $28^{\circ} \mathrm{C}$, but had no effect on crude protein digestibility. This may have implications for the role of carbohydrates as source of energy in practical diets for shrimp. The results also suggest that the fish meal used might have been of reduced quality.
\end{abstract}

Key words: Digestibility, temperature, salinity, soybean meal, fish meal, shrimp

\title{
RESUMEN
}

Se determinó la digestibilidad de la harina de soya y la harina de pescado en el camarón blanco (Litopenaeus vannamei) a dos diferentes temperaturas $\left(22^{\circ} \mathrm{C}\right.$ y $\left.28^{\circ} \mathrm{C}\right)$ y salinidades $(16 \%$ y $35 \%$ o), utilizando dietas preparadas con ingredientes disponibles en México. La digestibilidad de la harina de soya fue más alta comparada con la de la harina de pescado, en términos de digestibilidad aparente de materia seca, proteína cruda y aminoácidos, lo que sugiere que las proteínas de la harina de soya son más digeribles. Para la harina de pescado, la digestibilidad aparente de la materia seca fue menor en condiciones de mayor salinidad y $28^{\circ} \mathrm{C}$ de temperatura, mientras que la digestibilidad aparente de los carbohidratos se redujo en condiciones de elevada salinidad a ambas temperaturas. La salinidad elevada también tiene un efecto en la reducción de las digestibilidades aparentes de la materia seca, los lípidos y los carbohidratos de la harina de soya a $28^{\circ} \mathrm{C}$, pero no se encontró ningún efecto de ésta sobre la digestibilidad aparente de la proteína cruda. Esto puede tener implicaciones en el papel de los carbohidratos como un recurso energético adecuado en las dietas balanceadas para camarones. Los resultados también sugieren que la harina de pescado utilizada pudo haber sido de mala calidad.

Palabras clave: Digestibilidad, temperatura, salinidad, harina de soya, harina de pescado, camarón.

\section{INTRODUCTION}

Aquaculture production, especially that of carnivorous fishes and crustaceans, depends completely on the use of fish meal and fish oil as main sources of proteins and lipids. Today, there is a tendency to reduce this dependence through the use of other sources or alternatives with equal or similar nutritional quality, but lower cost (Tacon, 1997).

The accurate measurement of nutrient digestion and assimilation efficiency of the

\section{INTRODUCCIÓN}

La producción por acuacultura, especialmente de peces carnívoros y de crustáceos, depende del uso de la harina y el aceite de pescado como fuentes principales de proteína y lípidos. Hoy en día existe la tendencia a reducir ésta dependencia mediante el uso de otros recursos o fuentes alternativas de proteínas y lípidos, con igual o similar calidad nutritiva, pero de más bajo costo (Tacon, 1997).

La medición precisa de la digestión de los nutrientes y la eficiencia de asimilación de los 
dietary components, loosely termed digestibility, is essential to achieve an optimal diet formulation, both biologically and economically. Several researchers have derived feed digestibilities or assimilation efficiencies from growth experimental data (Fenucci et al., 1982; Bordner et al., 1983; Smith et al., 1985). Others have examined the metabolic effects of diet changes (Nelson et al., 1977; Clifford and Brick, 1979; Bordner et al., 1983). The digestive enzyme analysis is yet another analytical tool that has been used to evaluate digestion in marine crustaceans (Lee et al., 1984; Lan and Pan 1993; Ezquerra et al., 1998).

The direct measurement of the apparent digestibility of diets in shrimp is difficult because of problems when measuring total ingestion and egestion in an aquatic environment (Akiyama et al., 1989). However, previous digestibility studies on Litopenaeus vannamei have reported that diet and protein digestibility coefficients are influenced by different dietary protein sources as well as by the age of the animals studied (Smith et al., 1985). Seidman and Lawrence (1985) studied the effects of different dissolved oxygen (DO) levels and found that the apparent digestibilities of the total dry matter (DMAD) at concentrations of 1, 2, 3 and $4 \mathrm{mg} \mathrm{L}^{-1}$ were not significantly different.

Shiau et al. (1992) investigated the digestibility of fish meal, soybean meal and casein in Penaeus monodon in both brackish and seawater (16\%o and 32\%o of salinity, respectively). Salinity did not affect the digestibility of casein nor that of fish meal but it affected the digestibility of the soybean meal, which was significantly lower at 32\%o. Coelho (1984) examined the effects of salinity $(10 \%$ - $40 \%$ ) at a given componentes en una dieta, denominadas comúnmente digestibilidad, es esencial para lograr una formulación biológica y económicamente óptima. Algunos investigadores han estimado la digestibilidad de los alimentos o sus eficiencias de asimilación a partir de datos experimentales de crecimiento (Fenucci et al., 1982; Bordner et al., 1983; Smith et al., 1985). Otros han examinado los efectos metabólicos del cambio de dietas (Nelson et al., 1977; Clifford y Brick, 1979; Bordner et al., 1983). El análisis de las enzimas digestivas es también otra herramienta analítica que se utiliza para evaluar la digestión de los crustáceos marinos (Lee et al., 1984; Lan y Pan, 1993; Ezquerra et al., 1998).

La medición directa de la digestibilidad aparente de las dietas en los camarones es difícil por los problemas que representa medir en el medio acuático la ingesta total y las heces (Akiyama et al., 1989). Sin embargo, estudios previos de digestibilidad en Litopenaeus vannamei reportan que los coeficientes de digestibilidad de la dieta y de las proteína son influenciados por las diferentes fuentes protéicas de la dieta, así como por la edad de los animales estudiados (Smith et al., 1985). Seidman y Lawrence (1985) estudiaron los efectos de diferentes niveles de oxígeno disuelto (DO) y encontraron que las digestibilidades aparentes de la materia seca total (DAMS), a concentraciones de 1, 2, 3 y $4 \mathrm{mg} \mathrm{L}^{-1}$, no eran significativamente diferentes.

Shiau et al. (1992) investigaron la digestibilidad de la harina de pescado, la harina de soya y la caseina en Penaeus monodon tanto en agua salobre (16\%) como en agua de mar (32\%o). La salinidad no afectó la digestibilidad de la caseína ni la de la harina de pescado pero si afectó la digestibilidad de la harina de soya, la cual resultó significativamente menor a 32\%o. Coelho (1984) examinó los efectos de la salinidad (10\%o-40\%) a una cierta temperatura en $P$. vannamei y $P$. stylirostris en el 
temperature in $P$. vannamei and $P$. stylirostris in the laboratory. The results indicated that salinity had little effect but if low-protein feeds $(20 \%)$ were supplied, DMAD reduced by 30 to $40 \%$. The crude protein apparent digestibility (CPAD) was not affected. The shrimp showed higher DMAD, CPAD and aminoacid apparent digestibilitiy (AAAD) at the higher salinity when soybean meal ( $86 \%$ of the feed) was fed, but they were not affected with fish meal or casein at the same level.

Several researchers have examined the effects of temperature on feed digestibilities in crustacea. Temperature had no effect on the DMAD in Procambarus clarkii and Macrobrachium rosenbergii fed with a highprotein feed (Nose, 1964; Newman et al., 1982). CPAD was found to be affected by temperature in Penaeus monodon (Shen and Liu, 1993). Ezquerra et al. (1998) evaluated the CPAD at different temperatures in P. vannamei, using an in vitro $\mathrm{pH}$-stat assay and an enzyme preparation from shrimp hepatopancreas. Although temperature has been shown to influence the degree of hydrolysis of fish protein samples (Dimes and Haard, 1994), Ezquerra et al. (1998) could not demonstrate any differences among the hydrolysis degrees at 23,25 and $27^{\circ} \mathrm{C}$.

The present study was carried out in order to evaluate, in vivo, the apparent digestibility of protein, lipids, dry-matter and aminoacids of two diets in L. vannamei at different temperatures and salinities that harvesters use to find in their ponds. From this diets one was based on high-cost fish meal, and the other was based on low-cost soybean meal. laboratorio. Los resultados indicaron que la salinidad tuvo pocos efectos, a menos de que los alimentos tuvieran un bajo contenido protéico (20\% de proteína), en cuyo caso la DAMS se redujo en un 30 a un $40 \%$. La digestibilidad aparente de la proteína cruda (DAPC) no se vió afectada. Los camarones mostraron mayores digestibilidades aparentes de la materia seca, de proteínas crudas y de aminoácidos (DAAA) a salinidades más elevadas cuando se proporcionó harina de soya $(86 \%$ del alimento), pero éstos mismos factores no se vieron afectados cuando la harina de pescado o la caseína se ofrecieron al mismo nivel.

Varios investigadores han examinado los efectos de la temperatura sobre la digestibilidad de alimentos en los crustáceos. Nose (1964) y Newman et al. (1982) observaron que la temperatura no tenía efecto sobre la DAMS en Procambarus clarkii y Macrobrachium rosenbergii alimentados con una dieta alta en proteínas. Se encontró que, en Penaeus monodon, la DAPC era afectada por la temperatura (Shen y Liu, 1993). Ezquerra et al. (1998) evaluaron la DAPC a diferentes temperaturas en $P$. vannamei usando el método $\mathrm{pH}-$ stat y una preparación enzimática del hepatopáncreas de los camarones en un ensayo in vitro. Aunque se ha visto que la temperatura influencia el grado de hidrólisis de las muestras de proteína de pescado (Dimes y Haard, 1994), Ezquerra et al. (1998) no pudieron demostrar diferencias entre grados de hidrólisis a 23, 25 y $27^{\circ} \mathrm{C}$.

El presente estudio se llevó a cabo para comparar la digestibilidad aparente, in vivo, de la proteína, los lípidos, la materia seca y los aminoácidos de dos dietas en L. vannamei, a temperaturas y salinidades que los productores de camarón suelen encontrar en sus estanques de cultivo. De las dos dietas, una fue preparada a base de harina de pescado de alto costo $\mathrm{y}$, la otra, se hizo a base de soya, de más bajo costo. 


\section{MATERIALS AND METHODS}

\section{Experimental shrimp}

L. vannamei juveniles were obtained from a stock held in ponds at the La Cruz del Naranjero farm, Sinaloa, Mexico. All shrimp had been fed a maintenance ration of a commercial shrimp feed (approximately 35\% protein, dry pellet).

\section{Experimental diets}

Two isonitrogenous and isocalorific diets were formulated based on practical and technical-graded feedstuffs obtained from commercial sources. The feeds were prepared in the laboratory by drying, grinding and passing them through a $0.5-\mathrm{mm}$ screen mesh. A meat mincer with a 2.0 -mm-diameter die was used to produce the spaghetti-shaped diet which was cut in pieces and dried overnight at $40^{\circ} \mathrm{C}$. Then it was packed in plastic bags and stored in a freezer at $-20^{\circ} \mathrm{C}$, until required. The formulations and subsequent proximate analyses are shown in table 1.

\section{Proximate analysis}

Analyses for crude protein $(\% \mathrm{~N} \times 6.25)$, Kjeldahl nitrogen (Tecator 1030), total lipids, fibre, ash and moisture of the diets and the faecal samples were performed as described by the Association of Official Analytical Chemists (AOAC, 1984). The proximate analyses of the diets varied only slightly from the designed composition. The aminoacid composition (table 2) was determined after hydrolysis in $6 \mathrm{~N}$ $\mathrm{HCl}$ for $24 \mathrm{~h}$ at $105^{\circ} \mathrm{C}$ to $112^{\circ} \mathrm{C}$. The samples were evaporated to dryness, mixed with $\mathrm{Na}$ EFDR buffer and were then analyzed using a

\section{MATERIALES Y MÉTODOS}

\section{Camarones experimentales}

Los camarones juveniles de L. vannamei utilizados en el experimento se obtuvieron de la granja La Cruz del Naranjero, localizada en el estado de Sinaloa, México. Todos los camarones habían sido alimentados con una dieta comercial para camarón $(35 \%$ de proteína, pellet seco).

\section{Dietas experimentales}

Se formularon dos dietas isonitrogénicas e isocalóricas con ingredientes de alimentos balanceados y grado técnico obtenidos comercialmente. Los alimentos se prepararon en el laboratorio secando, moliendo y cerniéndolos a través de una malla de $0.5 \mathrm{~mm}$. Para producir la dieta en forma de espagueti, se utilizó un molino de carne con salidas de $2 \mathrm{~mm}$ de diámetro, los espaguetis secos se cortaron en pedazos, se secaron toda una noche a $40^{\circ} \mathrm{C}$, se empacaron en bolsas de plástico y después se almacenaron en un congelador $\mathrm{a}-20^{\circ} \mathrm{C}$, hasta ser utilizados. Las fórmulas y sus subsecuentes análisis proximales se muestran en la tabla 1 .

\section{Análisis proximal}

Los análisis de proteína cruda $(\% \mathrm{~N} \times 6.25)$, nitrógeno Kjeldahl (Tecator 1030), lípidos totales, fibras, cenizas y humedad de las dietas y las muestras fecales se realizaron de acuerdo a los métodos de la Asociación de Químicos Analíticos Oficiales (Association of Official Analytical Chemists; AOAC, 1984). Los análisis proximales de las dietas solo difirieron ligeramente de la composición diseñada. La composición de aminoácidos (tabla 2) se determinó por hidrólisis en $\mathrm{HCl} 6 \mathrm{~N}$ durante $24 \mathrm{~h}$ a $105^{\circ} \mathrm{C}-112^{\circ} \mathrm{C}$. Las muestras fueron secadas, se mezclaron con solución buffer Na-EFDR y después se analizaron utilizando un 
Table 1. Percent composition and proximate analysis of experimental diets.

Tabla 1. Formulación porcentual y análisis proximal de las dietas experimentales.

\begin{tabular}{|c|c|c|}
\hline Feed Ingredient & Fishmeal diet & Soybean diet \\
\hline Fish meal $^{\mathrm{a}}$ & 67.15 & 0.00 \\
\hline Soybean meal & 0.00 & 89.59 \\
\hline Fish oil & 0.82 & 5.94 \\
\hline Corn starch (pregelatinised) & 26.53 & 0.22 \\
\hline Squid meal ${ }^{\mathrm{b}}$ & 0.50 & 0.50 \\
\hline Vitamin mixture ${ }^{\mathrm{c}}$ & 0.50 & 0.50 \\
\hline Mineral mixture ${ }^{\mathrm{d}}$ & 0.50 & 0.50 \\
\hline Agar agar & 1.50 & 1.00 \\
\hline Carboxymethyl celulose & 1.00 & 0.50 \\
\hline Betaine & 0.50 & 0.25 \\
\hline Chromic oxide & 0.50 & 0.50 \\
\hline \multicolumn{3}{|l|}{ Proximate analysis $^{\mathrm{e}}$} \\
\hline Crude protein & 46.99 & 46.80 \\
\hline Total lipid & 8.46 & 6.87 \\
\hline Ash & 15.45 & 7.36 \\
\hline Fiber & 0.57 & 3.67 \\
\hline $\mathrm{NFE}^{\mathrm{f}}$ & 28.53 & 35.30 \\
\hline Chromic oxide & 0.48 & 0.49 \\
\hline Energy $^{\mathrm{g}}\left(\mathrm{kJ} \mathrm{g}^{-1}\right)$ & 19.33 & 19.83 \\
\hline $\mathrm{P}: \mathrm{E}\left(\mathrm{mg} \mathrm{kJ}^{-1}\right)^{\mathrm{h}}$ & 24.38 & 23.62 \\
\hline
\end{tabular}

Results are percentages, expressed on a dry matter basis.

${ }^{a}$ Menhaden fish meal (Zapata Industry, Ensenada, Baja California, Mexico).

${ }^{\mathrm{b}}$ Oven-dried 10 hours at $55^{\circ} \mathrm{C}$, laboratory-prepared.

${ }^{\mathrm{c}}$ Modified $\mathrm{BT}_{\mathrm{m}}$ salt mixture plus minerals (mg/kg-diet): zinc, 31.5; iodine, 4.8; selenium, 0.4; cobalt, 0.4 .

${ }^{\mathrm{d}}$ Vitamin (/kg-diet) mixture with: vitamin A, $80000 \mathrm{IU}$; vitamin $\mathrm{D}_{3}$, $2000 \mathrm{IU}$; vitamin E, $500 \mathrm{IU}$; vitamin K, $20 \mathrm{mg}$; niacin, $300 \mathrm{mg}$; riboflavin, $60 \mathrm{mg}$; pyridoxine, $57 \mathrm{mg}$; thiamin, $60 \mathrm{mg}$; panthothenic acid, $147 \mathrm{mg}$; biotin, $2 \mathrm{mg}$; folic acid, 20mg; vitamin $\mathrm{B}_{12}, 0.1 \mathrm{mg}$; choline chloride, $3000 \mathrm{mg}$; inositol, $200 \mathrm{mg}$; vitamin C, $500 \mathrm{mg}$.

${ }^{\mathrm{e}}$ Based on triplicate analysis; standard deviations were less than $1.0 \%$, except for protein $(<3 \%)$.

${ }^{\mathrm{f}}$ Nitrogen-free extract (calculated by difference).

${ }^{\mathrm{g}}$ Energy values as: protein, $23.6 \mathrm{~kJ} \mathrm{~g}^{-1}$; fat, $39.5 \mathrm{~kJ} \mathrm{~g}^{-1}$; and carbohydrate, $17.2 \mathrm{~kJ} \mathrm{~g}^{-1}$ (Aksnes et al., 1996).

${ }^{\mathrm{h}} \mathrm{P}: \mathrm{E}=$ protein to energy ratio (Jones and De Silva, 1997). 
Cabanillas-Beltrán et al.: Digestibilty of diets in Litopenaeus vannamei

Table 2. Aminoacid composition of experimental diets.

Tabla 2. Composición de aminoácidos de las dietas experimentales.

\begin{tabular}{|c|c|c|}
\hline Essential aminoacid & Fish meal & Soybean meal \\
\hline Threonine & 1.58 & 1.36 \\
\hline Valine & 1.51 & 1.28 \\
\hline Isoleucine & 1.26 & 1.20 \\
\hline Leucine & 2.27 & 2.22 \\
\hline Phenylalanine & 0.43 & 0.33 \\
\hline Histidine & 0.95 & 0.75 \\
\hline Lysine & 2.28 & 1.68 \\
\hline Arginine & 1.88 & 2.04 \\
\hline Methionine & 0.59 & 0.22 \\
\hline \multicolumn{3}{|c|}{ Non-essential aminoacids } \\
\hline Aspartic acid & 3.32 & 3.86 \\
\hline Serine & 1.49 & 1.83 \\
\hline Glutamic acid & 4.04 & 4.97 \\
\hline Proline & 1.53 & 1.56 \\
\hline Glycine & 1.98 & 1.33 \\
\hline Alanine & 1.69 & 1.25 \\
\hline Cystine & 0.24 & 0.74 \\
\hline Tyrosine & 1.21 & 1.54 \\
\hline
\end{tabular}

Data are averaged values from three replicas.

Results are expressed as dry-matter percent.

High Performance Amino Analyzer (Beckman System 6300). The tryptophan concentration was not determined. cromatógrafo de líquidos de alta presión (Beckman System 6300). La concentración de triptofano no fue determinada. 


\section{Experimental protocol}

The diets were tested at $16 \%$ and $35 \%$ of salinity, first at $22^{\circ} \mathrm{C}$ and then at $28^{\circ} \mathrm{C}$. Each treatment had three replicates distributed at random in two re-circulation systems for a total of 24 chambers. Each chamber was an adaptation of the Guelph method design (Martinez et $a l .$, 2001). Water was supplied to each experimental system after biological and mechanical filtration, temperature control $\left( \pm 1^{\circ} \mathrm{C}\right)$ and aeration. Daily measurements of temperature, salinity (Atago refractometer), $\mathrm{pH}$ (Corning $56 \mathrm{pH}$ meter) and dissolved oxygen (YSI 59 D.O.meter) were taken in tanks selected at random. Ammonia, nitrite and nitrate (Strickland and Parsons, 1977) were measured in four chambers randomly selected, twice a week. Dechlorinated tap water was added to the biofilters to replace evaporation losses, as required.

During the experiment, the chambers were covered with black net mesh to reduce the effects of the stress caused by the disturbance of the laboratory staff passing-by. Groups of 5 animals (10.2 $\pm 1.4 \mathrm{~g}$, mean weight) were selected at random for each diet treatment and then, they were transferred into the experimental arrays where they were acclimated during 8 days. Before each feeding procedure, all the fecal material was removed. The shrimp were fed ad libitum during all the experimental period twice a day, at 10:00 and 15:00 hours. The uneaten feed was removed from each chamber 60 minutes after feeding, and the feces in each chamber were collected from the settling trap into a sample bottle. Fecal samples were then gently rinsed with distilled water and dried overnight on petri dishes at $105^{\circ} \mathrm{C}$. The experiment lasted 15 days and samples were pooled for analysis.

\section{Protocolo Experimental}

Las dietas se probaron a $16 \%$ y $35 \%$ de salinidad, primero a $22^{\circ} \mathrm{C}$ y después a $28^{\circ} \mathrm{C}$. Cada tratamiento tuvo tres réplicas distribuídas al azar en dos sistemas de recirculación, para un total de 24 cámaras experimentales. Cada cámara fue un diseño adaptado del método de Guelph (Martínez et al., 2001). El agua fue suministrada a cada sistema experimental después de pasar por filtros mecánicos y biológicos, control de temperatura $\left( \pm 1^{\circ} \mathrm{C}\right)$ y aireación. Diariamente se midió temperatura, salinidad (refractómetro Atago), $\mathrm{pH}$ (potenciómetro Corning 56) y oxígeno disuelto (medidor de oxígeno YSI 59), en tanques seleccionados al azar. El amoniaco, los nitritos y los nitratos se midieron dos veces por semana en cuatro cámaras seleccionadas al azar dos veces por semana (Strickland y Parsons, 1977). Se añadió agua desclorurada a los biofiltros para remplazar el agua perdida por evaporación.

Durante el experimento, las cámaras se cubrieron con redes negras para reducir el efecto del estrés causado por el paso del personal de laboratorio. Se seleccionaron al azar grupos de 5 animales $(10.2 \pm 1.4 \mathrm{~g}$ de peso, en promedio) para cada dieta, transfiriéndose al sistema experimental en donde se aclimataron durante 8 días. Antes de cada alimentación, toda la materia fecal se eliminó del sistema. Los camarones se alimentaron ad libitum durante todo el periodo experimental dos veces al día a las 10:00 y a las 15:00 horas. El alimento no consumido se eliminó después de 60 minutos de cada alimentación y las heces de cada cámara se colectaron en una botella mediante las trampas de sedimentación. Las muestras fecales se enjuagaron con agua destilada y se secaron en cajas de petri durante 12 horas a $105^{\circ} \mathrm{C}$. El experimento duró 15 días, extrayendo muestras para el análisis. 


\section{Digestibility determination}

The apparent digestibility of the nutrients $(\% \mathrm{D})$ was determined with the formula described by Lovell (1989):

\section{Determinación de la digestibilidad}

La digestibilidad aparente de los nutrientes $(\% \mathrm{D})$ se determinó mediante la fórmula descrita por Lovell (1989):

$$
\% \mathrm{D}=\frac{\text { Amount of nutrient fed }- \text { Amount of nutrient in feces }}{\text { Amount of nutrient fed }} \times 100
$$

In order to evaluate this apparent digestibility, chromic oxide was added to the diets as an index of the undigested compound. The percentage of utilization is calculated from the concentrations of this index in the feed and the excreta (McGinnis y Casting, 1964; Hepher, 1993). Chromic oxide levels, both in diets and feces, were determined by first digesting the oganic matter with nitric acid and then oxidizing $\mathrm{Cr}_{2} \mathrm{O}_{3}$ to $\mathrm{Cr}_{2} \mathrm{O}_{7}$ with perchloric acid, followed by the colorimetric analysis of the dichromate ion with diphenylcarbazide (Furukawa and Tsukahara, 1966). All chemical determinations were made on a dry-weight basis and in triplicate. The apparent digestibility coefficients (ADC) of the nutrients, based on the indicator method, were calculated using the equation:
Con el fin de poder evaluar esta digestibilidad aparente, a las dietas se les añadió óxido de cromo como un indicador del componente no digerido. El porcentaje de utilización es calculado a partir de las concentraciones de este indicador en el alimento y en la excreta (McGinnis y Casting, 1964; Hepher, 1993). Los niveles de óxido de cromo en dietas y heces se determinaron mediante la digestión de la materia orgánica con ácido nítrico. Posteriormente se llevó a cabo la oxidación del $\mathrm{Cr}_{2} \mathrm{O}_{3}$ a $\mathrm{Cr}_{2} \mathrm{O}_{7}$ con ácido perclórico, seguida por el análisis colorimétrico del ión dicromato con difenilcarbazida (Furukawa y Tsukahara, 1966). Todas las determinaciones químicas se hicieron en base al peso seco y por triplicado. Los coeficientes de digestibilidad aparente (ADC) de los nutrientes, en base al método del indicador, se calcularon usando la ecuación:

$$
\operatorname{ADC}(\%)=100-100 \times\left(\frac{\% \mathrm{Cr}_{2} \mathrm{O}_{3} \text { in diet }}{\% \mathrm{Cr}_{2} \mathrm{O}_{3} \text { in feces }} \times \frac{\% \mathrm{Nfe}}{\% \mathrm{Nd}}\right)
$$

where Nfe is the amount of the nutrient in feces, and $\mathrm{Nd}$ is the amount of the nutrient in the diet (Forster and Gabbot, 1971).

The apparent digestibility of the energy (ADE) coefficient was calculated using the equation of Koshio et al. (1992): en donde Nfe es la cantidad del nutriente en las heces, y Nd es la cantidad de nutrientes en la dieta (Forster y Gabbot, 1971).

El coeficiente de digestibilidad aparente de la energía (ADE) se calculó utilizando la ecuación de Koshio et al. (1992):

$$
\mathrm{ADE}=1-\frac{\% \text { energy values in feces } / \% \mathrm{Cr}_{2} \mathrm{O}_{3} \text { in feces }}{\% \text { energy values in diet } / \% \mathrm{Cr}_{2} \mathrm{O}_{3} \text { in diet }} \times 100
$$




\section{Statistical analysis}

Percent data were transformed to the arcsine of their square-root prior to analysis. Differences in digestibility were assessed by analysis of variance. Multiple comparisons among means were made using Tukey's test (Montgomery, 1984).

\section{RESULTS}

Mean temperature, salinity, $\mathrm{pH}$, ammonia, nitrite and nitrate levels in the experimental systems during the studies are shown in table 3. All values were stable and within the accepted limits during the trials.

Proximate analyses (table 1) indicate no difference between the protein percent of both diets, lower total lipid content in soybean meal and high-ash and low-fiber content in fish meal. Nitrogen-free extract is higher in soybean meal while energy is similar in both diets.

The apparent digestibilities of proteins, dry-matter, lipids and carbohydrates in the experimental diets are shown in table 4 . The DMAD of soybean (65.7 to $72.9 \%$ ) was generally higher than that of fish meal $(56.7 \%$ to $65.2 \%$ ). However, with both protein sources, the digestibility at $28^{\circ} \mathrm{C}$ and $35 \%$ of salinity was significantly lower than in the other treatments. The CPAD was significantly higher for soybean meal (88.2 to $89.1 \%$ ) than for fish meal $(73.4$ to $76.2 \%)$ diets. However, both temperature and salinity had no effect on the CPAD of any of the diets tested. The apparent digestibility of lipids (LAD) in fish meal was higher at $28^{\circ} \mathrm{C}$, at both salinity levels, while for soybean meal it was only higher at $28^{\circ} \mathrm{C}$ and $16 \%$. There was no consistent pattern in the total carbohydrate apparent digestibility (CAD), although in soybean meal diets at $28^{\circ} \mathrm{C}$ and $35 \%$, it yielded significantly lower values.

\section{Análisis estadístico}

Los datos porcentuales obtenidos fueron transformados al arco seno de su raíz cuadrada antes de someterlos al análisis estadístico. Las diferencias en la digestibilidad se evaluaron mediante análisis de varianza. Para la comparación múltiple entre medias se utilizó la prueba de Tukey (Montgomery, 1984).

\section{RESULTADOS}

En la tabla 3 se muestran la temperatura media, la salinidad, el pH y los niveles de amoniaco, nitritos y nitratos en los sistemas experimentales durante el estudio. Todos los valores se mantuvieron estables y dentro de los límites aceptables para la especie durante las pruebas.

Los resultados del análisis proximal (tabla 1) indican que no hay diferencia en el porcentaje de proteína de las dos harinas, que la harina de soya es más baja en lípidos totales y que la harina de pescado es más alta en cenizas y más baja en fibras. El extracto libre de nitrógeno es mayor en la harina de soya mientras que la energía es similar en ambas dietas.

Las digestibilidades aparentes de proteínas, materia seca, lípidos y carbohidratos de las dietas experimentales se muestran en la tabla 4. La DAMS de la soya (de 65.7 a $72.9 \%$ ) fue generalmente más alta que la de la harina de pescado (de 56.7 a 65.2\%). Sin embargo, con ambas fuentes protéicas, la digestibilidad fue significativamente más baja a $28^{\circ} \mathrm{C}$ y $35 \%$ de salinidad que en los otros tratamientos. La DAPC fue significativamente más alta para la harina de soya (de 88.2 a $89.1 \%$ ) que para las dietas con harina de pescado (de $73.4 \%$ a $76.2 \%$ ). Sin embargo, la temperatura y la salinidad no tuvieron efecto sobre la digestibilidad de la proteína de ninguna de ambas harinas. La DAL fue mayor a $28^{\circ} \mathrm{C}$ con ambas salinidades en la harina de pescado, mientras que para la harina de soya, solamente fue mayor a $28^{\circ} \mathrm{C} \mathrm{y}$ 
Cabanillas-Beltrán et al.: Digestibilty of diets in Litopenaeus vannamei

Table 3. Water quality parameters in the system during the trials.

Tabla 3. Parámetros de calidad del agua del sistema durante los bioensayos.

\begin{tabular}{lccccc}
\hline Parameter & $n$ & System 1 & System 2 & System 3 & System 4 \\
\hline Salinity (\%o) & 30 & $16 \pm 1.0$ & $35 \pm 0.5$ & $16 \pm 1.0$ & $35 \pm 1.0$ \\
Temperature $\left({ }^{\circ} \mathrm{C}\right)$ & 30 & $22 \pm 0.9$ & $22 \pm 0.9$ & $28 \pm 1.0$ & $28 \pm 1.0$ \\
PH & 15 & $8.4 \pm 0.29$ & $8.6 \pm 0.34$ & $8.3 \pm 0.23$ & $8.2 \pm 0.16$ \\
Dissolved oxygen $\left(\mathrm{mg} \mathrm{L}^{-1}\right)$ & 15 & $5.9 \pm 0.82$ & $5.8 \pm 0.55$ & $6.3 \pm 0.52$ & $6.1 \pm 0.41$ \\
Ammonia $\left(\mathrm{mg} \mathrm{L}^{-1}\right)$ & 5 & $0.002 \pm 0.0001$ & $0.003 \pm 0.0001$ & $0.003 \pm 0.0007$ & $0.004 \pm 0.0001$ \\
Nitrite $\left(\mathrm{mg} \mathrm{L}^{-1}\right)$ & 5 & $0.002 \pm 0.0001$ & $0.001 \pm 0.0008$ & $0.002 \pm 0.0001$ & $0.001 \pm 0.0007$ \\
Nitrate $\left(\mathrm{mg} \mathrm{L}^{-1}\right)$ & 5 & $7.8 \pm 0.92$ & $7.5 \pm 0.93$ & $12.23 \pm 1.3$ & $11.77 \pm 1.2$ \\
\hline
\end{tabular}

Data are mean values \pm 1 standard deviation.

$n=$ number of observations.

Table 4. Apparent digestibility of protein, dry matter, lipids and carbohydrates in the experimental diets based on two protein sources, by Litopenaeus vannamei at two temperatures and two salinities.

Tabla 4. Digestibilidad aparente de proteína, materia seca, lípidos y carbohidratos las dietas experimentales basadas en dos fuentes protéicas, en Litopenaeus vannamei, a dos temperaturas y dos salinidades.

\begin{tabular}{lcccccccc}
\hline $\begin{array}{l}\text { Treatment/ } \\
\text { Constituent }\end{array}$ & \multicolumn{3}{c}{ Fish meal } \\
\hline $\begin{array}{l}\text { Temperature }\left({ }^{\circ} \mathrm{C}\right) \\
\text { Salinity (\%) }\end{array}$ & 22 & 22 & 28 & 28 & 22 & 22 & 28 & 28 \\
Dry matter & 16 & 35 & 16 & 35 & 16 & 35 & 16 & 35 \\
Protein & $65.2^{\mathrm{b}}$ & $64.8^{\mathrm{b}}$ & $60.2^{\mathrm{b}}$ & $56.7^{\mathrm{c}}$ & $72.6^{\mathrm{a}}$ & $72.9^{\mathrm{a}}$ & $74.7^{\mathrm{a}}$ & $65.7^{\mathrm{b}}$ \\
Lipids & $74.9^{\mathrm{b}}$ & $76.2^{\mathrm{b}}$ & $74.1^{\mathrm{b}}$ & $73.4^{\mathrm{b}}$ & $88.2^{\mathrm{a}}$ & $89.1^{\mathrm{a}}$ & $88.7^{\mathrm{a}}$ & $88.7^{\mathrm{a}}$ \\
Carbohydrates & $87.7^{\mathrm{b}}$ & $83.5^{\mathrm{b}}$ & $95.6^{\mathrm{a}}$ & $96.5^{\mathrm{a}}$ & $81.5^{\mathrm{b}}$ & $86.7^{\mathrm{b}}$ & $92.7^{\mathrm{a}}$ & $74.6^{\mathrm{c}}$ \\
\hline
\end{tabular}

In any row, values with the same superscripts are not significantly different $(P<0.05)$. 
Table 5. Apparent digestivility of the energy (ADE) in the experimental diets.

Tabla 5. Digestibilidad aparente de la energía (ADE) en las dietas experimentales.

\begin{tabular}{lccccc}
\hline Ingredient & $\begin{array}{c}\text { Temperature } \\
\left({ }^{\circ} \mathrm{C}\right)\end{array}$ & $\begin{array}{c}\text { Salinity } \\
(\%)\end{array}$ & $\begin{array}{c}\text { Gross Energy } \\
\left(\mathrm{kJ} \mathrm{g}^{-1}\right)\end{array}$ & $\begin{array}{c}\text { ADE } \\
\text { coefficient }(\%)\end{array}$ & $\begin{array}{c}\text { ADE } \\
\left(\mathrm{kJ} \mathrm{g}^{-1}\right)\end{array}$ \\
\hline Fish meal & 22 & 16.0 & 19.33 & $77.4^{\mathrm{b}} \pm 1.4$ & $14.96^{\mathrm{b}} \pm 0.2$ \\
& 22 & 35.0 & 19.33 & $77.2^{\mathrm{b}} \pm 1.3$ & $14.91^{\mathrm{b}} \pm 0.4$ \\
& 28 & 16.0 & 19.33 & $79.1^{\mathrm{b}} \pm 0.2$ & $15.28^{\mathrm{b}} \pm 0.1$ \\
& 28 & 35.0 & 19.33 & $77.9^{\mathrm{b}} \pm 0.9$ & $15.07^{\mathrm{b}} \pm 0.6$ \\
Soybean meal & 22 & 16.0 & 19.83 & $82.6^{\mathrm{a}} \pm 1.5$ & $16.37^{\mathrm{a}} \pm 0.3$ \\
& 22 & 35.0 & 19.83 & $83.2^{\mathrm{a}} \pm 1.1$ & $16.50^{\mathrm{a}} \pm 0.5$ \\
& 28 & 16.0 & 19.83 & $80.5^{\mathrm{a}} \pm 0.9$ & $15.49^{\mathrm{b}} \pm 0.3$ \\
& 28 & 35.0 & 19.83 & $77.0^{\mathrm{b}} \pm 1.2$ & $15.27^{\mathrm{b}} \pm 0.7$ \\
\hline
\end{tabular}

Data are averaged values from 3 replicates, \pm 1 standard deviation.

In any column, values with the same superscripts are not significantly different $(P<0.05)$.

ADE coefficients were generally higher for soybean meal $(77.0$ to $83.2 \%)$ than for fish meal diets (77.2 to $79.1 \%$ ), except for the soybean meal diet at the high-temperature and high-salinity treatment (table 5).

All of the 17 aminoacids studied (table 6) had significantly different digestibilities in the two protein sources, except for cystine, in fish meal at $28^{\circ} \mathrm{C}$ and in soybean meal at $22^{\circ} \mathrm{C}$ and for fenilalanine in which the lowest value was observed in soybean meal at $28^{\circ} \mathrm{C}$. The AAAD of soybean meal was, in general, greater than that of the fish meal.

\section{DISCUSSION}

The digestibility of soybean meal was consistently higher compared to that of fish meal, in terms of DMAD, CPAD and AAAD, suggesting that soybean meal proteins are more 16\%o. No hubo un patrón consistente en la digestibilidad aparente de carbohidratos (DAC), no obstante que, en la dieta de harina de soya a $28^{\circ} \mathrm{C}$ y $35 \%$, dio valores significativamente más bajos.

Los coeficientes de ADE fueron generalmente más altos para la harina de soya (de 77.0 a $83.2 \%$ ) que para las dietas con harina de pescado (de 77.2 a $79.1 \%$ ), con excepción del tratamiento que incluyó la dieta con harina de soya a la temperatura y la salinidad más altas (tabla 5).

De los 17 aminoácidos estudiados (tabla 6), todos tuvieron digestibilidades significativamente diferentes entre las dos fuentes protéicas, con excepción de la cistina, para la harina de pescado a $28^{\circ} \mathrm{C}$ y para la harina de soya a $22^{\circ} \mathrm{C}$, y de la fenilalanina en la que el valor más bajo fue observado en la harina de soya a $28^{\circ} \mathrm{C}$. La DAAA de la harina de soya fue en general mayor que la de la harina de pescado. 
Cabanillas-Beltrán et al.: Digestibilty of diets in Litopenaeus vannamei

Table 6. Apparent digestibility of aminoacids in the experimental diets, by shrimp Penaeus vannamei, at different temperatures and $35 \%$ of salinity.

Tabla 6. Digestibilidad aparente de los aminoácidos de las dietas experimentales, en el camarón Litopenaeus vannamei a diferentes temperaturas y $35 \%$ de salinidad.

\begin{tabular}{|c|c|c|c|c|}
\hline \multirow{2}{*}{$\begin{array}{l}\text { Ingredient } \\
\text { Temperature }\end{array}$} & \multicolumn{2}{|c|}{ Fish meal } & \multicolumn{2}{|c|}{ Soybean meal } \\
\hline & $28^{\circ} \mathrm{C}$ & $22^{\circ} \mathrm{C}$ & $28^{\circ} \mathrm{C}$ & $22^{\circ} \mathrm{C}$ \\
\hline \multicolumn{5}{|c|}{ Essential aminoacids } \\
\hline Threonine & $79.5^{\mathrm{b}} \pm 1.2$ & $81.0^{\mathrm{b}} \pm 1.4$ & $87.2^{\mathrm{a}} \pm 0.7$ & $88.8^{\mathrm{a}} \pm 1.9$ \\
\hline Valine & $79.5^{\mathrm{b}} \pm 1.1$ & $81.6^{\mathrm{b}} \pm 1.7$ & $88.8^{\mathrm{a}} \pm 1.4$ & $88.8^{\mathrm{a}} \pm 2.6$ \\
\hline Isoleucine & $83.2^{\mathrm{b}} \pm 1.5$ & $83.2^{\mathrm{b}} \pm 1.8$ & $90.5^{\mathrm{a}} \pm 2.0$ & $91.0^{\mathrm{a}} \pm 1.6$ \\
\hline Leucine & $82.9^{\mathrm{b}} \pm 1.4$ & $83.1^{\mathrm{b}} \pm 1.9$ & $89.9^{\mathrm{a}} \pm 1.4$ & $90.6^{\mathrm{a}} \pm 0.7$ \\
\hline Phenylalanine & $83.9^{\mathrm{b}} \pm 1.2$ & $84.4^{\mathrm{b}} \pm 1.6$ & $73.1^{\mathrm{c}} \pm 0.9$ & $96.3^{\mathrm{a}} \pm 1.8$ \\
\hline Histidine & $85.0^{\mathrm{b}} \pm 1.3$ & $85.9^{\mathrm{b}} \pm 1.5$ & $91.0^{\mathrm{a}} \pm 1.7$ & $90.6^{\mathrm{a}} \pm 0.8$ \\
\hline Lysine & $86.7^{\mathrm{b}} \pm 1.3$ & $87.0^{\mathrm{b}} \pm 2.3$ & $92.2^{\mathrm{a}} \pm 2.2$ & $92.3^{\mathrm{a}} \pm 1.7$ \\
\hline Arginine & $78.8^{\mathrm{b}} \pm 1.1$ & $81.6^{\mathrm{b}} \pm 1.5$ & $96.5^{\mathrm{a}} \pm 0.7$ & $94.7^{\mathrm{a}} \pm 0.5$ \\
\hline Methionine & $84.6^{\mathrm{b}} \pm 0.8$ & $92.2^{b} \pm 0.4$ & $94.2^{\mathrm{a}} \pm 0.6$ & $96.2^{\mathrm{a}} \pm 0.5$ \\
\hline \multicolumn{5}{|c|}{ Non-essential aminoacids } \\
\hline Aspatic acid & $77.2^{\mathrm{b}} \pm 1.8$ & $79.5^{\mathrm{b}} \pm 1.2$ & $91.4^{\mathrm{a}} \pm 0.4$ & $89.9^{\mathrm{a}} \pm 0.8$ \\
\hline Serine & $73.3^{\mathrm{b}} \pm 1.6$ & $77.3^{\mathrm{b}} \pm 1.1$ & $88.4^{\mathrm{a}} \pm 2.8$ & $88.1^{\mathrm{a}} \pm 1.9$ \\
\hline Glutamic acid & $78.7^{\mathrm{b}} \pm 1.8$ & $81.3^{\mathrm{b}} \pm 1.3$ & $92.7^{\mathrm{a}} \pm 1.3$ & $90.9^{a} \pm 0.9$ \\
\hline Proline & $71.4^{\mathrm{b}} \pm 0.7$ & $72.8^{\mathrm{b}} \pm 1.2$ & $89.7^{\mathrm{a}} \pm 1.4$ & $88.9^{\mathrm{a}} \pm 1.8$ \\
\hline Glycine & $66.5^{\mathrm{b}} \pm 0.9$ & $68.3^{\mathrm{b}} \pm 1.0$ & $86.6^{\mathrm{a}} \pm 1.6$ & $85.9^{\mathrm{a}} \pm 1.7$ \\
\hline Alanine & $76.2^{\mathrm{b}} \pm 0.8$ & $76.9^{\mathrm{b}} \pm 0.6$ & $89.5^{\mathrm{a}} \pm 1.7$ & $89.4^{\mathrm{a}} \pm 1.3$ \\
\hline Cystine & $84.3^{\mathrm{b}} \pm 0.8$ & nd & $96.2^{\mathrm{a}} \pm 0.9$ & $79.8^{\mathrm{b}} \pm 2.1$ \\
\hline Tyrosine & $74.6^{\mathrm{b}} \pm 1.1$ & $77.9^{\mathrm{b}} \pm 1.6$ & $91.2^{\mathrm{a}} \pm 1.5$ & $89.8^{a} \pm 1.9$ \\
\hline
\end{tabular}

Values are averaged values from three replicas (10/replica).

In any row, values with the same superscripts are not significantly different $(P<0.05)$.

nd $=$ not detected. 
easily digested. Compared to the values reported by Akiyama et al. (1989) for L. vannamei, the DMAD values obtained in this work for the fish meal were similar, while those obtained for soybean meal were consistently higher; the CPAD values obtained for fish meal were lower, while those for soybean were considerably higher. Above all, these results suggest that the fish meal used might have been of a low quality.

The DMAD for fish meal was reduced by the high salinity at $28^{\circ} \mathrm{C}$, whereas the $\mathrm{CAD}$ was reduced by the high salinity at both temperatures. High salinity also reduced the dry matter, the lipids and the carbohidrates' apparent digestibilities of soybean meal at $28^{\circ} \mathrm{C}$, but had no effect on CPAD, as found by De Silva and Perera (1984) for Tilapia mozambica. This suggests that, under the experimental conditions, $16 \%$ of salinity was better for the digestibility of the nutrients measured.

Although there have been few studies concerning the effects of temperature on the digestibility in crustaceans, it is likely that water temperature is an important variable. Although water temperature had a significant effect on feed ingestion, neither Nose (1964) nor Newman et al. (1982), in Procambarus clarkii and Macrobrachium rosenbergii, respectively, found significant differences in their DMAD. However, Shen and Liu (1993) found that in $P$. monodon CPAD was optimum at $30^{\circ} \mathrm{C}$ and fell rapidly at $35^{\circ} \mathrm{C}$. In respect to the temperatures used in the present study, which are commonly found in shrimp farming, these showed no influence in the CPAD as there was no significant difference between the values found for both meals. The CPAD was significantly lower at high temperature and high salinity in both meals, meaning that under these conditions the digestibility efficiency is lower, as was also observed in fish

\section{DISCUSIÓN}

La digestibilidad de la harina de soya, en términos de DAMS, DAPC y DAAA, fue más alta comparada con la de la harina de pescado, lo que sugiere que las proteínas de la primera son más fácilmente digeribles. En comparación con los valores obtenidos por Akiyama et al. (1989) para L. vannamei, los valores obtenidos en el presente estudio para la DAMS en la harina de pescado fueron similares, mientras que los valores obtenidos para la harina de soya fueron consistentemente más altos; los valores de DAPC para la harina de pescado fueron más bajos, mientras que los de la harina de soya fueron considerablemente más altos. Sobre todo, estos resultados sugieren que la harina de pescado utilizada puede haber sido de baja calidad.

Para la harina de pescado, la DAMS se vió reducida por la alta salinidad a $28^{\circ} \mathrm{C}$ y la DAC se redujo con las salinidades más elevadas, a ambas temperaturas. La salinidad más alta también redujo las digestibilidades aparentes tanto de la materia seca como de los lípidos y los carbohidratos de la harina de soya a $28^{\circ} \mathrm{C}$, pero no tuvo efectos sobre la DAPC, como encontraron De Silva y Perera (1984) en Tilapia mozambica. Lo anterior indica que, en las condiciones experimentales de este trabajo, una salinidad de $16 \%$ fue mejor para la digestibilidad de los nutrientes medidos.

A pesar de que ha habido pocos estudios con relación a los efectos de la temperatura sobre la digestibilidad en crustáceos, es importante reconocer que la temperatura es una variable importante. Ni Nose (1964), en Procambarus clarkii, ni Newman et al. (1982), en Macrobrachium rosenbergii, encontraron diferencias significativas en las respectivas DAMSs, a pesar de que en ambas especies la temperatura del agua tuvo un efecto significativo sobre la ingestion de alimento. Sin 
(Knutsen and Salvanes, 1999; Peres and OlivaTeles, 1999).

The AAAD in fish meal was significantly lower than in soybean meal, at both temperatures, which can be explained by the low quality of the fish meal protein due to a deficient quality control process. The results of the proximate analysis show that fish meal is higher in ashes, suggesting high levels of bones and scales, characteristics of a low-quality fish meal. Such a quality resulted in lower digestibility values.

In conclusion, in most of the cases, the digestibility indicators were lower for fish meal than for soybean meal.

\section{ACKNOWLEDGEMENTS}

This study was supported by CIAD, A.C. Mazatlan Unit. The technical assistance of Irma Martínez and Blanca González is acknowledged. LGR thanks for the support of the Department for International Development of the Government of the United Kingdom .

\section{REFERENCES}

Aksnes, A., Hjertnes, T. and Opstvedt, J. (1996). Comparison of two assay methods for determination of nutrient and energy digestibility in fish. Aquaculture, 140: 343-359.

Akiyama, D.M., Coelho, S.R. and Lawrence, A.L. (1989). Apparent digestibility of feedstuff by the marine shrimp Penaeus vannamei Boone. Nippon Suisan Gakkaishi, 55(1): 91-98.

AOAC. (1984). Official Methods for Analysis of the Association of Official Analytical Chemists, 14th. edition. Arlington, VA, $1141 \mathrm{pp}$.

Bordner, C.E., D'Abramo, L.R. and Conklin, D.E. (1983). Assimilation of nutrients by culture hybrid lobster (Homarus sp.) fed experimental diets. Journal of the Mariculture Society 14: 1124. embargo, Shen and Liu (1993) encontraron en P. monodon que la DAPC fue óptima a $30^{\circ} \mathrm{C} \mathrm{y}$ disminuyó rápidamente a $35^{\circ} \mathrm{C}$. En relación a las temperaturas usadas en el presente trabajo, las cuales son comunes en las granjas de camarón, éstas no mostraron influencia en la DAPC dado que no hubo diferencia significativa entre los valores encontrados en ambas harinas. La DAPC fue significativamente menor a alta temperatura y a alta salinidad con ambas harinas, lo cual significa que bajo estas condiciones existe menor eficiencia en la digestibilidad, como también se ha encontrado en peces (Knutsen y Salvanes, 1999; Peres y OlivaTeles, 1999).

La DAAA fue significativamente más baja en la harina de pescado que en la de soya a ambas temperaturas. Esto puede deberse a que la proteína de la harina de pescado era de mala calidad debido un deficiente control en el proceso de elaboración. Por otro lado, los resultados del análisis proximal indican que la harina de pescado tiene mayor porcentaje de cenizas, lo que sugiere altos niveles de escamas y huesos, indicativos de una harina de baja calidad. Dicha calidad influyó en los bajos resultados de digestibilidad.

En conclusión, en la mayoría de los casos los indicadores de digestibilidad para la harina de pescado fueron más bajos que para la harina de soya.

\section{AGRADECIMIENTOS}

Este estudio fue apoyado por CIAD, A.C. Unidad Mazatlán. Agradecemos la asistencia técnica de Irma Martínez y Blanca González. LGR agradece el apoyo del Departamento para el Desarrollo Internacional del Gobierno del Reino Unido.

Traducido al español por Adrián R. López. 
Clifford, H.C. III and Brick, R.W. (1979). A physiologycal approach to the study of growth and bioenergetics in the freshwater shrimp Macrobrachium rosenbergii. Proceedings of the World Mariculture Society, 10: 701-719.

Coelho, S.R. (1984). Effects of environmental salinity and dietary protein levels on digestibility in four species of penaeid shrimp. Master's Thesis, Texas A\&M University, College Station, Texas, USA.

De Silva, S.S. and M.K. Perera. (1984). Digestibility in Sarotherodon niloticus fry: Effect of dietary protein level and salinity with further observations on variability in daily digestibility. Aquaculture, 38: 293-306.

Dimes, L.E. and Haard, N.F. (1994). Estimation of protein digestibility I. Development of an in vitro method for estimating protein digestibility in salmonids (Salmo gairdneri). Comparative Biochemistry and Physiology 108A: 349-362.

Ezquerra, J.M., García-Carreño, F.L. and Carrillo, O. (1998). In vitro digestibility of dietary protein sources for white shrimp (Penaeus vannamei). Aquaculture, 163: 123-136.

Fenucci, J.L., Fenucci, A.C., Lawrence, A.L. and Zein-Eldin, Z.P. (1982). The assimilation of protein and carbohydrate from prepared diets by the shrimp, Penaeus stylirostris. Journal of the World Mariculture Society, 13: 134-145.

Forster, J. and Gabbott, P. (1971). The assimilation of nutrients from compounded diets by the prawns Palaemon serratus and Pandalus platyceros. J. Mar. Biol. Ass. U.K., 51: 943-961.

Furukawa, H. and Tsukahara, H. (1966). On the acid digestion methods for the determination of chromic oxide as an index substance in the study of digestibility of fish feed. Bull. Japan. Soc. Sci. Fish., 32(6): 502-508.

Hepher, B. (1993). Nutrición de Peces Comerciales en Estanques. Grupo Noriega (Editores), 406 pp.

Jones, P.L. and De Silva, S.S. (1997). Influence of differential movement of the marker chromic oxide and nutrients on digestibility estimations in the Australian freshwater crayfish Cherax destructor. Aquaculture, 154: 323-336.

Knutsen, I. and Salvanes, A.G.V. (1999). Temperature-dependent digestion handling time in juvenile cod and possible consequences for prey choice. Mar-Ecol-Prog-Ser 181: 61-79.

Koshio, S., Castell, J.D. and O'Dor, R.K. (1992). The effect of different dietary energy levels in crab-protein-based diets on digestibility, oxygen consumption, and ammonia excretion of bilaterally eyestalk-ablated and intact juvenile lobsters Homarus americanus. Aquaculture, 108:

85-297.

Lan, C.C. and Pan, B.S. (1993). In vitro digestibility simulating the proteolysis of feed protein in the midgut gland of grass shrimp (Penaeus monodon). Aquaculture 109: 59-70.

Lee, P.G., Smith, L.L. and Lawrence, A.L. (1984). Digestive proteases of Penaeus vannamei relationship between enzyme activity, size and diet, Aquaculture 42: 225-239.

Lovell, T. (1989). Nutrition and Feeding of Fish. Van Nostrand-Reinhold, New York, 260 pp.

Martínez-Palacios, C.A., Cabanillas-Beltrán, H., Ponce-Palafox, J.T., Chávez Sánchez, Ma,C. and Ross, L.G. (2001). A modified chamber designed for estimation of digestibility in shrimp. North American Journal of Aquaculture, 63: 252-255.

McGinnis, A.J. and Kasting R. (1964). Colorimetric analysis of chromic oxide used to study food utilization by phytophagous insects. Agricultural and Food Chemistry, 12: 259-262.

Montgomery, D.C. (1984). Design and Analysis of Experiments. Second Edition. John Wiley \& Sons. New York, 538 pp.

Nelson, S.G., Li, H.W. and Knight, A.W. (1977). Calorie, carbon and nitrogen metabolism of juvenile Macrobrachium rosenbergii (De Man) (Crustacea, Palaemonidae), with regard to trophic position. Comparative Biochemistry and Physiology 58A: 319-327.

Newman, M.W., Lutz, P.L. and Snedaker, S.C. (1982). Temperature effects on feed ingestion and assimilation efficiency of nutrients by the Malaysian prawn, Macrobrachium rosenbergii (De Man). Journal of the World Mariculture 
Cabanillas-Beltrán et al.: Digestibilty of diets in Litopenaeus vannamei

Society, 13: 95-103.

Nose, T. (1964). Protein digestibility of several test diets in cray and prawn fish. Tansui-ku Suisan Kenkyujo Kenkyu Hokoku, 14: 23-28.

Peres, H. and Oliva-Teles, A. (1999). Influence of temperature on protein utilization in juvenile European seabass (Dicentrarchus labrax). Aquaculture 170, (3-4): 337-348.

Seidman, E.R. and Lawrence, A.L. 1985. Growth, feed digestibility and proximate body composition of juvenile Penaeus vannamei and Penaeus monodon grown at different dissolved oxygen levels. Journal of the World Mariculture Society 16: 333-346.

Shen, X. and Liu, Y. (1993). The study of protein, fat and starch digestibilities of prawn (Penaeus orientalis). Collection of Oceanographic Works / Haiyang Wenji, 16: 76-82.

Shiau, S.Y., Lin, K.P. and Chiou, C.L. (1992). Digestibility of different protein sources by
Penaeus monodon raised in brackish water and in sea water. Journal of Applied Aquaculture. Aquaculture. 1: (3), 47-54.

Smith, L.L., Lee, P.G., Lawrence, A.L. and Strawn, K. (1985). Growth and digestibility by three sizes of Penaeus vannamei Boone: effects of dietary protein level and protein source. Aquaculture, 46: 85-96.

Strickland, J.D.H. and Parsons, T.R. (1977). A Practical Handbook of Seawater Analysis. Fisheries Research Board of Canada, Ottawa, $310 \mathrm{pp}$.

Tacon, A.G.J. (1997). Feeding tomorrow's fish: Keys for sustainability. In: Proceedings of the workshop of the CIHEAM Network on Technology of Aquaculture in the Mediterranean (TECAM). CIHEAM, FAO and IEO. Mazarrón (Spain) 24-26 June, 1996. pp. 11-33. 